\title{
FRANCISCO DE HINOJOSA Y SU ENTRADA A LA "MONTAÑA DE LOS MOXOS" (COCHABAMBA-BOLIVIA, SIGLO XVI)
}

\author{
FRANCISCO DE HINOJOSA'S INCURSION INTO THE "MOXOS \\ MOUNTAIN" (COCHABAMBA - BOLIVIA, $16^{\text {th }}$ CENTURY)
}

WaLter SÁNCHEZ

En los últimos años, la evidencia arqueológica ha comenzado a mostrar la presencia de caminos prehispánicos que articulaban el altiplano, los valles, los yungas situados en la cordillera oriental de los Andes ("montaña") y la Amazonía. Muchos de estos caminos fueron inhabilitados durante el Período Colonial temprano generándose la idea de que este bloque cordillerano fue una suerte de escollo que dividía las tierras altas de las tierras bajas (la Amazonía). El artículo aborda el ingreso de Francisco de Hinojosa hacia la llamada "montaña de los Moxos" y su fracaso por llegar a los llanos de Moxos. Asimismo, pone en evidencia que el cierre de estos antiguos caminos, fue una decisión consciente de las sociedades de los yungas, posiblemente en un intento de cortar sus vínculos con la sociedad colonial.

Palabras clave: Bolivia, Cochabamba, yungas, Francisco de Hinojosa, caminos coloniales.

In recent years, archaeological evidence has begun to show the presence of pre-Hispanic roads that connected the altiplano, valleys, the Yungas (a zone of tropical valleys) in the eastern Andes and the Amazon basin. Many of these roads were abandoned during the Early Colonial Period, leading to the idea that this mountain range was an obstacle that separated the Altiplano highlands from the Amazon lowlands. This article addresses Francisco de Hinojosa's incursion into the so-called "Moxos Mountain" and his failure to reach the Moxos plains. It also shows that the closure of these ancient roads was apparently a conscious decision made by the societies inhabiting the Yungas, possibly in an attempt to sever their ties with the colonial society.

Keywords: Bolivia, Cochabamba, yungas, Francisco de Hinojosa, colonial roads.

\section{INTRODUCCIÓN}

El presente texto es parte del proyecto de investigación arqueológica "Buscando Caminos", cuyo objetivo es abordar, a partir de la documentación temprana, las posibles rutas de interacción prehispánicas entre los valles, la puna (denominada en documentos de los siglos XVI-XVII como cordillera de Moxos), los yungas o sub-andino (la montaña de los Moxos) y los llanos amazónicos (los llanos de los Moxos) en CochabambaBolivia y el poblamiento en los yungas. ${ }^{1}$ A partir de este conocimiento documental, se quiere abordar, en una segunda fase, el trabajo arqueológico. Por lo tanto, el objetivo de este artículo es analizar la entrada de Francisco de Hinojosa a las montañas de los Moxos, en la hipótesis de que el análisis de este documento podría arrojar pistas sobre los lugares de ingreso y las poblaciones que la habitaban. Sobre este último punto, existe evidencia documental de la presencia de grupos que habitaban los yungas de Chuquiuma, Aripucho, Incachaca y posiblemente Tablas Monte, y que son hetero-denominados como Umu/Amo/Yumu. Fuentes locales del siglo XVI y XVII dan cuenta de la presencia de gente Yuroma que confinaba con "los llanos é tierras de los indios Mojos é Chunchos que están conjuntos y

A Walter Sánchez, Instituto de Investigaciones Antropológicas y Museo Arqueológico-Universidad Mayor de San Simón, e-mail:walteryambae@hotmail.com

Recibido: diciembre 2015. Aceptado: agosto 2017. 
límites de la Cordillera de esta Villa [de Oropeza] [...] [y] de que había grandes minerales de oro y plata", así como grupos llamados Rache (Soruco 1899: 82; Saignes 1985a, 1985b). Las fuentes documentales sugieren en todos ellos la existencia de una suerte de verticalidad (territorial y de uso de recursos) que se habría desplegado entre la Amazonía y los valles de Cochabamba (Sánchez 2008, Schramm 2012).

A partir del testimonio arqueológico, muchos investigadores han reportado evidencia de ocupación de larga data en el sub-andino (yungas) (cf. Alconini 2016), así como de importantes interacciones entre las tierras bajas y los Andes (Alconini 2013; Cruz 2014; Alconini 2016). En Cochabamba, tales interacciones son evidentes desde los trabajos de Brockington et al. (1995) para el Período Formativo, Céspedes (2001) y Sánchez (2008) para el Horizonte Medio, Intermedio Tardío e Inca (Sánchez 2008, 2011). De hecho, para el caso de los yungas de Incachaca/Paracti, Tablas Monte y San José/San Julián no solo existe evidencia arqueológica de constantes articulaciones e interacciones entre los valles y los llanos aluviales del Chapare ya para el Horizonte Medio (Cf. Céspedes 2001; Sánchez 2008, 2011, 2012), sino de caminos formalmente construidos (Sánchez 2008). De la misma manera, han sido reportados caminos formalmente construidos, de clara factura inca, en los yungas de Chuquiuma (Pereira \& Céspedes 1982), Arepucho, Icuna y Antahuagana (cf. Pereira \& Céspedes 1982; Sánchez 2008) y el rio San Jacinto. Dichos caminos llegaban posiblemente hasta los llanos amazónicos del Chapare, aunque hasta hoy no se ha realizado un reconocimiento sistemático debido a las dificultades operativas de trabajar dentro de una zona de bosque alto. En el caso de los Caminos Incas (Inca yan), hay que señalar que siempre conducen a complejos arqueológicos con filiación cronológica que se remonta al Horizonte Medio e incluso al Formativo y con evidencia material local proveniente de los valles y de la Amazonía. ${ }^{2}$ Este hecho pondría en evidencia que los incas usaron y remodelaron caminos construidos mucho antes de su llegada.

El énfasis puesto en la búsqueda de trazas de conexión entre los valles y la Amazonía es importante debido a que la arqueología boliviana ha separado tradicionalmente las tierras altas (la parte andina) de las tierras bajas (Amazonía y Chaco) como si fueran dos estancos separados y no interconectados. Bajo esta mirada, la cordillera oriental y el sub-andino (los yungas) ha sido entendida como un escollo que separó dos espacios y, consecuentemente, dos sociedades y civilizaciones. Tal comprensión ha conducido, incluso, a una suerte de especialización académica entre arqueólogos dedicados a las tierras altas (los Andes) y otros a las bajas (Amazonas/Chaco). Si bien, como un primer paso, investigadores como Saignes (1985b) habían llamado la atención desde la etnohistoria sobre la necesidad de encarar el estudio histórico de aquellos espacios olvidados por la historiografía como de aquellos que se ubicaban en la vertiente oriental de los Andes, su estudio es reciente desde la arqueología, aunque con una fuerte concepción de la cordillera como "frontera" (para una relación sobre algunos trabajos tanto arqueológicos como etnohistóricos véase Pärssinen \& Siiriänen 2003, del Río 2011, Alconini 2013).

Los valles de Cochabamba, ubicados entre los 2000 - $3000 \mathrm{msnm}$, son encajonados dentro de la cordillera Oriental, destacando el valle Bajo, el valle Central (donde se ubica la actual ciudad de Cochabamba), el valle de Sacaba y el valle Alto. Otros valles más pequeños, pero importantes, son el de Pocona, Mizque y Totora. Los valles se caracterizan por su clima templado con una media de $23^{\circ} \mathrm{C}$. El bloque cordillerano, hacia el norte, está conformado por una extensa puna entre los 3500 a $4000 \mathrm{msnm}$, con cumbres por encima de los $5000 \mathrm{msnm}$. Poseen un clima frío y seco. El sub-andino o la cara oriental de esta cordillera, donde se ubican los yungas o la montaña, es una extensa cadena montañosa que baja abruptamente desde los $4000 \mathrm{msnm}$ hasta los 350 msnm, nivel en el que su ubican los llanos amazónicos del Chapare. Un elemento que hay que destacar de la montaña de los Moxos (los yungas) es que constituye una zona de gran pluviosidad y está dominada por un bosque alto de neblina, con registros que van de los 4000 a los $9000 \mathrm{~mm}$ por año (figs. 1 y 2).

\section{Algunos datos de la vida del Capitán Francisco de Hinojosa ${ }^{3}$}

Urquidi (1949: 61) destaca la presencia de Francisco de Hinojosa a principios de la década de 1570, como un "vecino de la ciudad de la Plata". Este dato hace suponer que Hinojosa tenía por entonces su residencia en Sucre y que, desde ahí, comenzaba a expandir sus negocios, articulando zonas agropecuarias (Chuquisaca y Cochabamba) con el mercado minero de Potosí, por entonces el centro económico colonial más importante de los Andes. 


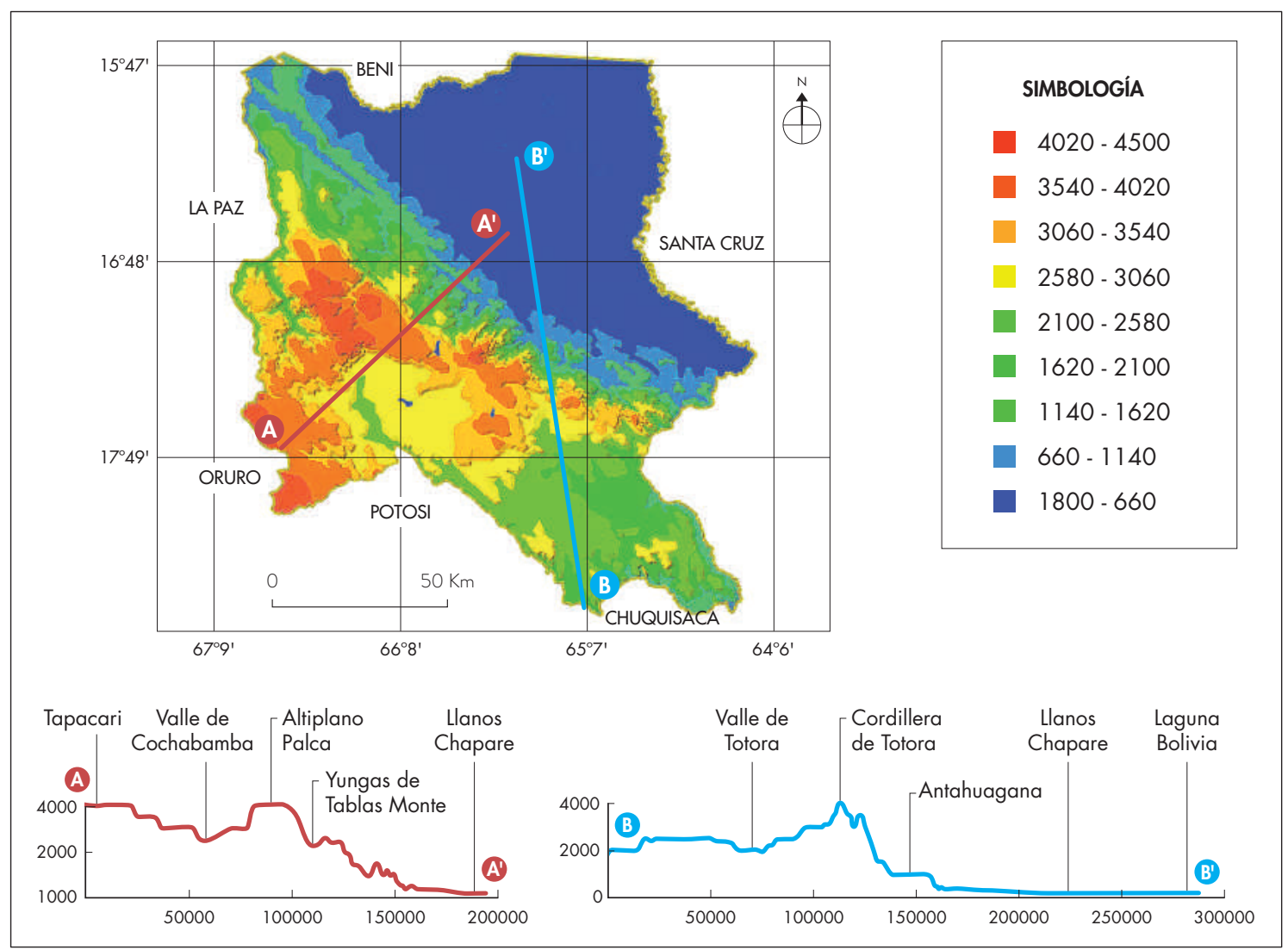

Figura 1. Departamento de Cochabamba con dos cortes transversales altitudinales que muestran los valles, la puna (altiplano), los yungas y la amazonía. Figure 1. Department of Cochabamba with two altitudinal cross sections, showing the valleys, the puna (Andean high plateau), the Yungas zone and the Amazon basin.



Figura 2. Cochabamba. Precipitación pluvial según zonas eco-regiones. Figure 2. Cochabamba. Rainfall by eco-regions. 
En 1571, por medio de una carta-poder llevada por Baltasar Gonzales (su mayordomo), pide ser admitido como poblador de la recientemente fundada villa de Oropeza (actual ciudad de Cochabamba). Además, solicita tierras y solares:

Sepan quantos esta Carta de Poder vieren como yo Francisco de Hinojosa, residente que al presente soy en este Asiento de Canata del Valle de Cochabamba, jurisdicción de la Villa de Oropesa, otorgo y conozco por esta presente Carta a vos Baltasar Gonzáles que sois presente, pa que por mi nombre y como yo mismo podays pedir y pidays sea admitido en esta Villa de Oropesa, que nuevamente se funda en este Valle de Cochabamba, por vez ino della e me den tierras y solares e las demas que dan a los demas y vecinos y pobladores de la dicha Villa de tomar y aprehender la posesion e posesiones de los tales solares e tierra e demas cosas en mi nombre y las continuar y hazer todo aquello que yo podria hazer y cerca dello y de lo a ello anexo e dependiente, siendo necesario parecer en contienda de juicio parezcays ante el Capitán Grmo. Osorio, Corregidor de la dicha Villa, a quien esta cometida la dicha población (Urquidi 1949: 111).

Por otros documentos se sabe que Hinojosa ya tenía actividades agropecuarias en el valle de Cochabamba. Una carta de hipoteca otorgada en 1571 a Juan Serrano Dávalos por el préstamo de dinero -saldado en 1573- muestra que desarrollaba una intensa actividad agropecuaria:

E pa que mas seguro esteis de la paga de lo suso dicho vos hipoteco por especial y expresa hipoteca Myll cabezas de puercos chicos y grandes, que tengo en este Asiento de Canata y la sementera que ANSI mesmo tengo en este Valle de Cochabamba en Companya de Francisco de Orellana, los cuales dichos puercos y sementera que ansi vos hypoteco me obligo de no lo vender ni enagenar en manera alguna (Urquidi 1949: 109-110).

Otra de las actividades empresariales en las que se hallaba embarcado era el comercio de harina de maíz entre Cochabamba y Potosí, tal como muestra la siguiente Carta de Poder, hecha en 1571:

Otorgo y conozco por esta presente Carta que doy y otorgo todo my poder cumplido, libre, llenero y bastante, a vos Po de las Cassas, presente, especialmente para que por mi y en mi nombre y como yo mismo, representando mi propia persona podays, en la villa de Potosí, donde van cargados trescientes carneros de la dicha tierra mios de harina de mais villcaparo, lo vender. E ansi mismo otras trescientas cargas que los indios de la Encomienda de Francisco de Orellana, vecino de la ciudad de La Plata, me estan obligados a llevar a la dicha Villa, vender y vendays la dicha comida al fiado o contado como os pareciere. E del valor de la dicha comida y no alcanzando de los dichos trescientos carneros que ansi myos van cargados, os hazer pago y cobrar un myll y cien pesos de plata corriente, que yo os debo y estoy obligado a vos pagar, por una escriptura que contra my teneys de mayor quantia, de plazo pasado [...] (Urquidi 1949: 112). ${ }^{4}$

Resumiendo estos dos documentos, podemos destacar los siguientes bienes agrícolas y pecuarios de Hinojosa: "trescientos carneros de la dicha tierra mios" (llamas), "Myll cabezas de puercos chicos y grandes" y "sementera en este Valle de Cochabamba en Companya" del encomendero Francisco de Orellana.

Todas sus actividades eran administradas por un mayordomo, Baltasar Gonzales, "con el qual se ha sentado aquí [ilegible] y a el se le ha dado lo que ha sido a su cargo, hasta el dia de hoy, ansi de coca, como de trigo, mayz, puercos, vacas e ovejas e ganado de la tierra" (Urquidi 1949: 113).

\section{La Entrada a las "montañas de los Moxos" y la repartición de tierras}

Se sabe que, en la década de 1570, Hinojosa fue corregidor de Cochabamba, momento en el que hace reparticiones de tierras a españoles en los valles Central y de Sacaba. ¿Cuál fue el motivo para tales reparticiones? Un hecho que parece haber incentivado a los residentes españoles la "repartición de tierras" en Cochabamba era la supuesta constatación de que, "en todo el dicho Valle de Cochabamba no hay naturales, sino todos son mitimaes de diferentes partes, los quales puso alli el Inca, que partió y dividió las tierras del dicho Valle por suios y suertes, repartiéndolos a los dichos mitimaes, según la cantidad de gente, que cada uno tenia" (Urquidi 1949: 93). Dicho de otra manera, al ser los valles de Cochabamba tierras ganadas por el Inca tras someter a los grupos locales, le correspondía ahora, por derecho de conquista, a la Corona española. Esta era, sin embargo, una "verdad" a medias, ya que existían grupos locales que tenían tierras antes de la llegada de los Incas como los Chuy, Quta, Qhawi y Sipe Sipe. De hecho, uno de estos grupos, los Qhawi vinculados a los Quta de Pocona, elevarán a la Audiencia y a la Cancillería Real de la ciudad de La Plata una demanda. En efecto, en febrero de 1587 se presentan ante el capitán Fernando de Casorla Narváez, maese de campo, corregidor y justicia mayor y juez de naturales del Cabildo de Oropeza, don Pedro Cato y don Francisco Moroco, caciques principales y de los indios Qawi "que rresiden en el balle de sacaba" 
pertenecientes al Repartimiento de Pocona. Ambos caciques denuncian:

Francisc ${ }^{\circ}$ de hinojosa siendo corregidor en esta billa por complacer y tener gratos a ciertos soldados para llebarlos a la jornada de los moxos hiço sierta repartición de tierras en este balle en tres personas quel parecio y quiso como fueron joan garcia pinto e joan de sanabria y a sus hijos en el dicho balle de sacaba y en el de clissa e a otras muchas personas en nuestra tierras que habemos y tenemos de tiempo inmemorial a esta parte (AнмC.ECC. Vol. 9, № 1, 28.IX.1611).

Vistos los alegatos, este caso será resuelto con la devolución de las tierras a los Qawi:

su alteça proveyendo justicia dio por ningunas las dichas reparticiones de tierras fechas por el dicho francisco de hinojosa en las dichas nuestras tierras y mando a pedro quiros de abila nuestro corregidor que fue del partido de misque y el dio comision para que personalmente viniese a este balle y en cenos metiese y amparase en las dichas nuestras tierras lansando dellas a las personas que estuviesen en ellas y pretendiesen tener algun derecho a ellas por la dicha repartición [...] en virtud de la qual el dicho nuestro corregidor dio posesion e poseciones de las dichas nuestras tierras como todo ello consta y paresse (AHMC.ECC. Vol. 9, No 1, 28.Ix.1611).

En otros casos, muchas tierras consideradas "vaças", pertenecientes a grupos altiplánicos y que antes habían sido entregadas por el Inca dentro los "repartimientos de tierras”, serán consolidadas por los soldados españoles que habían participado en la entrada hacia los Moxos. Tal el caso, por ejemplo, de Pedro Ireviño, español, quién señala en su testamento: “//4 [...]. - Yten declaro que yo tengo titul.o [...] de la [...] tierra de la puna de guara guara y [ilegible] de molino que me dio e rrepartio el cap. Francisco de Hinojosa que el fue poblador en esta villa de Cochabamba" (ANB-EC 1612.7).

Estos ejemplos muestran los cambios y los conflictos que se venían produciendo en los valles de Cochabamba producto de: a) el abandono que cientos de mitmaqkunas que habían llegado con la presencia inca; b) la consolidación de tierras por parte de los grupos que eran reconocidos como "originarios", es decir, residentes antes de la llegada inca y; c) el cambio en el régimen de la tierra por parte de los españoles en este caso, como "pago" por ingresos hacia zonas hasta ese entonces desconocidas.

\section{La Entrada a la montaña de los Moxos llamada también "de Cochabamba"}

Es poco lo que se conoce acerca de las diversas entradas realizadas por los primeros pobladores hispanos en los valles de Cochabamba hacia las montañas llamadas de los Mojos. El historiador Thierry Saignes (1985a: 6364) ha destacado las siguientes (ver Tabla 1 más abajo).

Una entrada importante fue realizada por el capitán Francisco de Hinojosa con la venia del virrey Toledo y el patrocinio de la Audiencia de Charcas. Aparentemente, este ingreso fue realizado en 1582, aunque, como veremos más adelante, Hinojosa habría realizado entradas anteriores, a partir de las cuales convenció a las autoridades coloniales de que gente que "es rica y mucha", se hallaba más allá de las montañas (fig. 3).

Tabla 1. Entradas españolas hacia las "montañas de Moxos (siglos XVI-XVII). Table 1. Spaniards incursions to "montañas de Moxos" (16 $6^{\text {th }}$ and $17^{\text {th }}$ centuries)

\begin{tabular}{c|c|c|c|c|c} 
FECHAS & RESPONSABLES & ÁREA/GRUPOS & RESULTADO & OTRAS PRECISIONES & FUENTES \\
\hline 1564 & Alemán & Montaña de Cochabamba/Yuroma & EMMI & Desaparición & RGI 1 \\
\hline 1569 & Cuellas/Ortega & Cochabamba-Río Yuroma & EMIR & Expedición no autorizada & Sarmiento \\
\hline $1582(?)$ & F. de Hinojosa & Montaña de Cochabamba & EMIR & Expedición autorizada. Fracaso & RGI 2 \\
\hline 1588 & Angulo & Montaña de Cochabamba & EMIR & Maurtúa (Ed.) 9 \\
\hline 1630 & Bolívar & Montaña de Cochabamba & EMIR & Desaparición de franciscanos & Mendoza
\end{tabular}

$\mathrm{EE}=$ Entrada de Evangelización $\mid \mathrm{EM}=$ Entrada Militar $\mid \mathrm{IR}=$ Información/retorno $\mid \mathrm{MI}=$ Muerto por los indios 


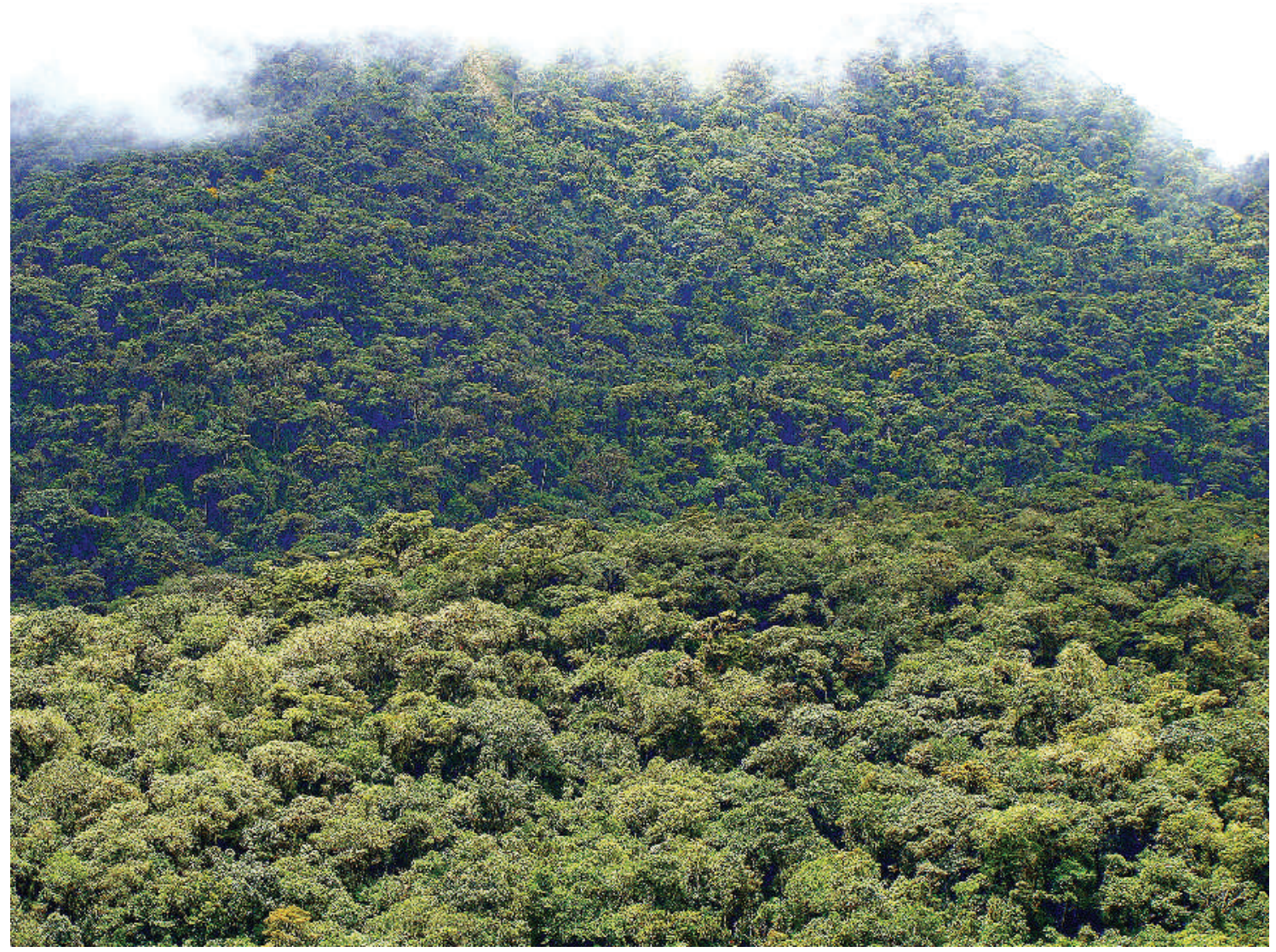

Figura 3. Fragmento de paisaje de los yungas de Cochabamba en la parte denominada "montaña de los Moxos". Se halla cubierta por un bosque denso alto de neblina constante (fotografía de Walter Sánchez). Figure 3. Landscape of the Yungas (tropical valleys) of Cochabamba known as the "Moxos Mountain", covered in dense, continually mist-shrouded forest (photo by Walter Sánchez).

Según la "Relación de lo sucedido en la Entrada de los Mojos" que Hinojosa hace al virrey Martín Enríquez, señala que: "a quien se debe desta jornada [es al] Sr. Virrey D. Francisco de Toledo". De hecho, sabemos que el pedido formal de ingreso fue elevado a la Real Audiencia de La Plata y esta entidad lo elevó a instancias superiores.

Con respecto a la relación y a la entrada, La Audiencia de la Plata emitió un informe enviado a Lima, en el que se detalla las condiciones en las que debía entrar:

al capitan Hynojosa se le dieron por mi recaudos y provisiones para la entrada que auia de hazer por Cochabamba con las limitaciones e ynstrucion y advertencias que V.Sa. havra visto en prevención de lo que rrefiere V.Sa. es la suya con que no lleue consigo delinquentes ninguno lo qual mandan a V.S.a que ansi se ejecute y guarde que a el se le escreue haga su entrada como le esta mandado que en quanto a lo de los ingleses que se auisa que venian con designio de poblar esta (actividad) no parece que ay siendo tan lejos de quererme ay donde y principalmente en Potosí se que ay mucha gente vagamunda y lo mismo me dize V.Sa. y que conbiene desaguarla a lo qual se atendio atención en la data de la licencia para esta entrada (1580 (fs. 2) во АВNB, ALP, CAch-15).

La relación de esta jornada hecha por Francisco de Hinojosa, muestra aspectos de su organización y las dificultades para llevar adelante la expedición, tal como hace constar este documento:

y porque desee servir a S. M. en esta jornada me la hizo, y ANSI yo con el celo y cuidado que a S. M. se debe, hice la gente en esta provincia, $y$ hecha $y$ habiendo gastado muchos dineros en adereçar lo necesario y comprar arcabuces y otros peltrechos; $y$ al tiempo y cuando la gente comenzaba a caminar e yo habia de partir de esta ciudad, el D.or. Barros, 


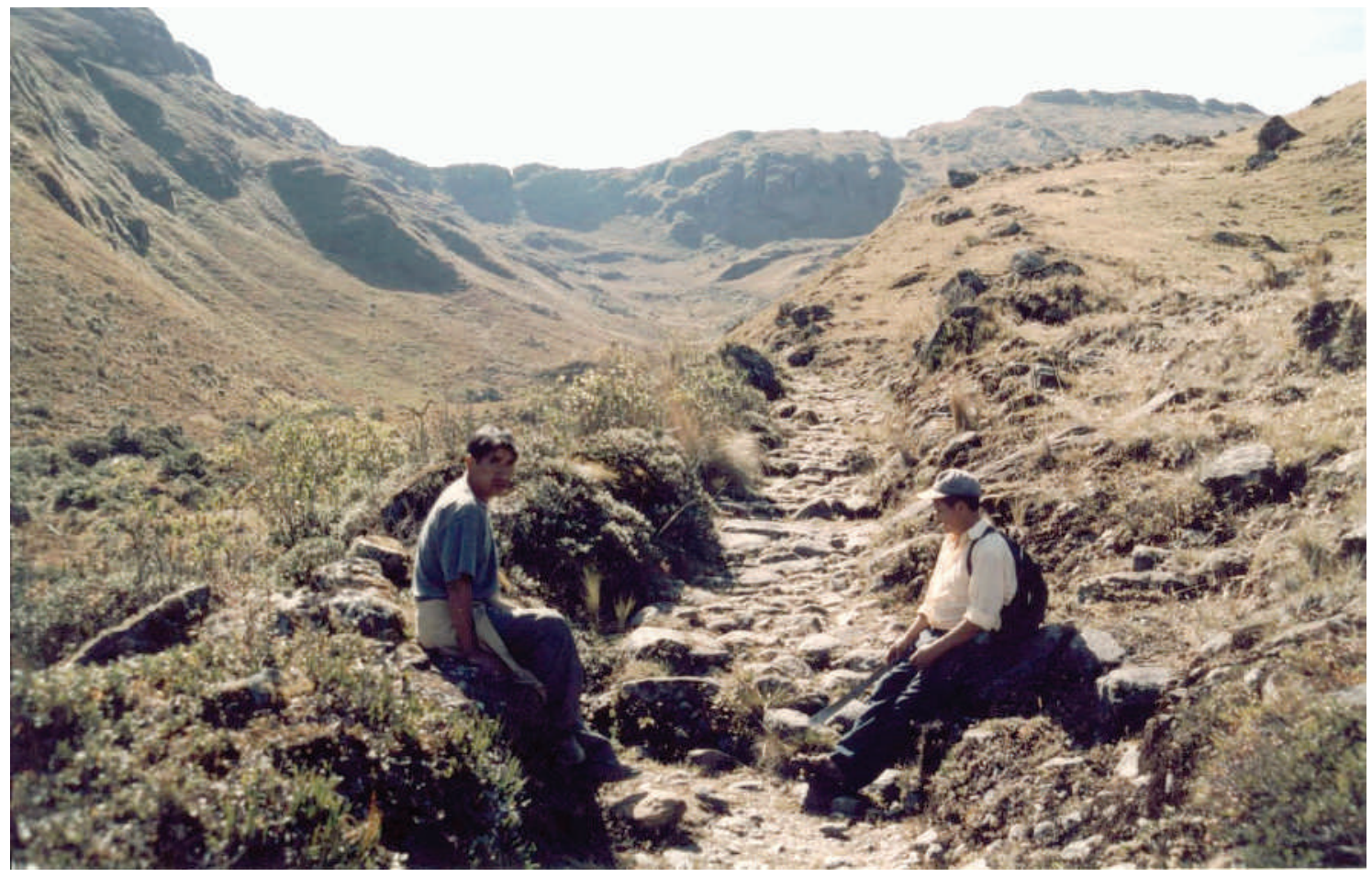

Figura 4. Fragmento de camino empedrado inca. Ramal Colomi-Incachaca (fotografía de Walter Sánchez C.). Figure 4. Segment of a paved Inca road along the Colomí-Incachaca branch (photo by Walter Sánchez C.).

que presidia, me impidio y deshizo la mejor gente que hasta hoy para servir a S.M. se ha juntado en el Peru y se fue cada uno por su parte, donde me llevaron muchos arcabuces y otras cosas y ceso el hacer de la jornada e yo perdi mas de 20 mil pesos que solo de mi parte habia gastado, sin lo que antes de esto gaste cuando entre a descubrir esta tierra, que todo ha sido a mi costa con harto trabajo de mi persona, de donde he quedado con algunas enfermedades. (1580 (fs. 2) BO ABNB, ALP, CACh-15)

Aquí vemos que Hinojosa hizo una entrada anterior en la que descubre estas tierras. El dato se solventa mucho más cuando continúa la Relación:

y luego escribi y di cuenta al Sr. Virrey, el cual mando que no se me pusiese impedimento alguno, y asi procure de volver a rehacer lo que he podido, y sabe dios con cuanto trabajo y costa hice hasta 30 hombres; y para que estos se fuesen recogiendo y juntando en un valle 24 leguas la tierra adentro, donde yo tenia de paz unos pocos indios que alli hay, que son tan pocos que no llegan a veinte. (1580 (fs. 2) во ABNB, ALP, CAch-15).

No sabemos a ciencia cierta por dónde penetró Hinojosa y hasta dónde llegó. Dos rutas son probables, debido a la existencia de caminos prehispánicos: a) hacia la puna de Colomi con dirección hacia el río Chapare; b) hacia la puna de Altamachi, con destino al río Secure, por donde se señala que vivían los Yuroma, y c) hacia el río Cotacajes (llamado también Quetoto) y que desembocaba en el río Beni (fig. 4).

En su recorrido señala no haber llegada a los llanos de los Moxos, sino a un valle (dentro de la montaña), situado a 24 leguas tierra adentro (¿de los yungas?), y donde había unos pocos indios. Para tal efecto, continúa escribiendo:

Envie delante a Pedro Velez de Guevara por cabeza de los que se fuesen juntando y para que alli hiciese sementeras para poder conseguir lo de adelante y que estuviese alli hasta que se juntase la copia de gente que el senor Virrey me dio por comision, y que siendo esto junto ya, entrara. Y por algunos malos tratamientos se huyeron estos indios, $y$ el Pedro Velez de Guevara levanto de alli con esta ocasión sin orden mia ni hacermelo saber, y fue a un valle que esta la tierra adentro 20 leguas, donde hay poblados algunos indios, aunque pocos.

Aunque no sabemos cuáles son los "pocos indios" con los que se encuentran en los valles yungueños, 


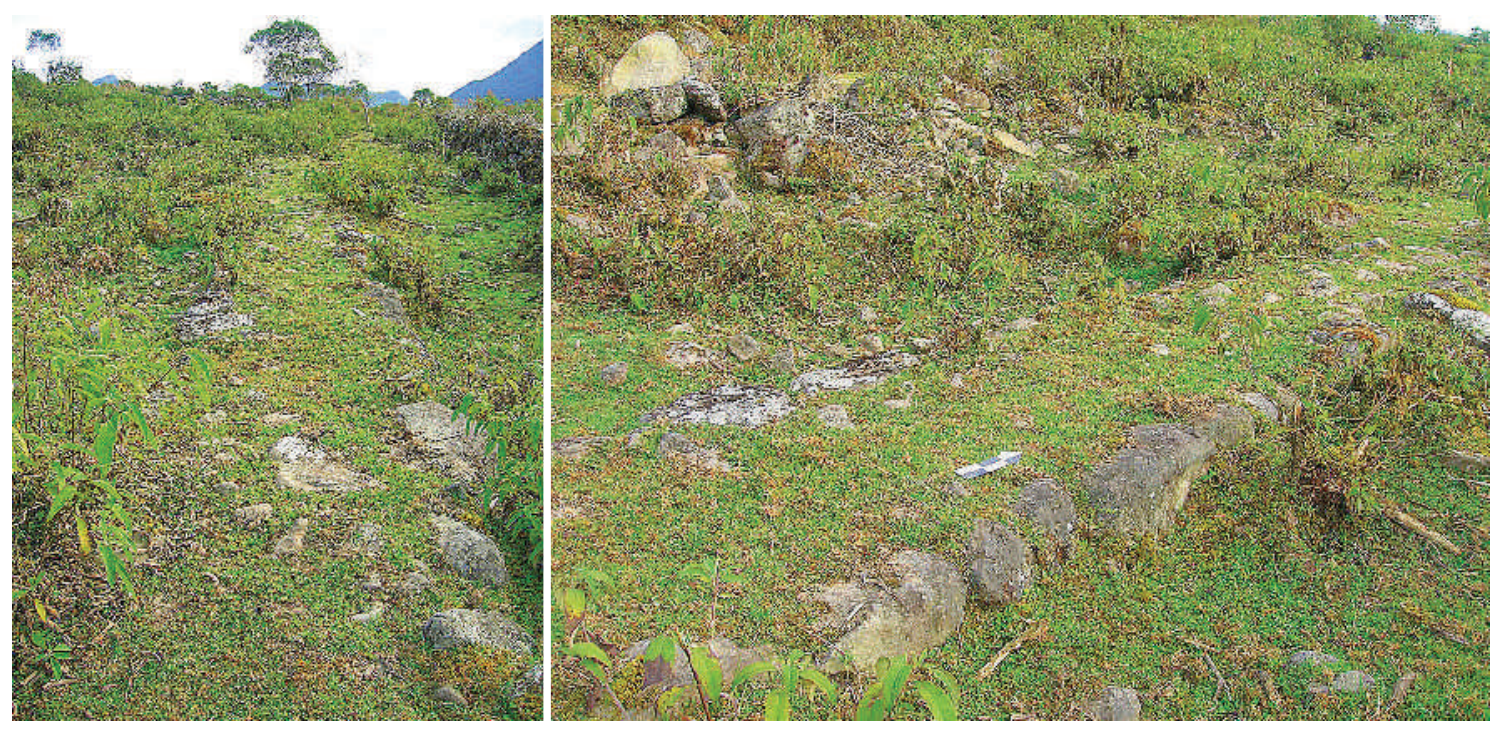

Figura 5. Fragmentos de camino empedrado inca en Incachaca. Cochabamba (fotografía de Walter Sánchez C.). Figure 5. FSegment of a paved Inca road along the Colomí-Incachaca branch (photo by Walter Sánchez C.).

es posible lanzar tres hipótesis: 1) que haya sido un grupo local (¿Umu/Amo/Yumu o Yuroma o Rache?, de posible filiación lingüística Yuracare?); 2) indios de los valles templados de Cochabamba, huidos luego de la conquista hispana o 3) antiguos mitmaqkuna que se quedaron en la montaña.

No hay ninguna evidencia de que se tratara de "indios de guerra", aunque muestran tácticas recurrentes entre los yuracare de los siglos XVIII y XIX, como la huida y el "griterío": "y a mi me dieron guaçabara cuando fui a descubrir y vi ser pocos y de poca fuerza; porque demas de correr yo aquellos valles con 25 hombres que tenia e ser la mitad muchachos, no me hicieron ningun daño ni tienen fuerça para ello, y solo un aracabuz o dos que se dispararon resistio toda la furia.".

El dato de poca gente y el hecho de que este grupo de montaña no tenía vínculos con los de tierras bajas, generan también la imagen de que podría haber sido un grupo de mitmaqkunas residentes en la montaña: "Y la gente de que se tiene la noticia, que es rica y mucha, esta al principio de la tierra de estos 30 leguas, o poco mas, y estos que digo donde Pedro Veles fue no tienen comunicación ni trato con los otros, que son unos indiezuelos que están recogidos en la Montaña”.

De lo señalado, se colige que el grupo no pudo pasar los bosques cerrados de los yungas y llegar hasta los llanos amazónicos (fig. 5).
Luego del fracaso para alcanzar los llanos aluviales conocidos como "de Moxos" y la imposibilidad de consolidar un pueblo en los yungas para continuar la expedición, el Virrey del Perú, en una carta enviada a la Audiencia de la Plata (1.9.1582), es firme al prohibir nuevas entradas debido a los problemas que causaban.

vino una información hecha en la Villa de Cochabamba sobre el daño que sestendio hauer receuido los de la entrada de los Mojos y diome harta pena hasta que por las otras dos de V.S [...].entendi hauer salido todos de saluamento y que el [...] fue despoblar el e como fue despoblar el pueblo que hauian poblado y venirse que no lo tengo por muy grande respecto a que era poca gente, fin orden ni concierto de permanecer y de pressente no me parece de que hay que prouer en ello sino solo a perceuir a francis.co de Hinojosa que si ha de hacer la entrada ha de ser por su per.sona y por lo menos con sesenta hombres bien aderaçados y preuenidos de lo necessari.o como que lo dexe V. S.a sera seruido mandar que así se lo notiffique, y no dexalle entrar desta manera, ni consentille que ponga Teniente por tan poco tuuo comisión para nombralle la otra vez, y con acsque de que yba a recoger la gente mientras quel entraua se estuuo hasta agora y según la mala orden que deuiron de tener no ha sido poco no ubiedecerles mas Dmo. V.S. no consienta que entre mas gente fin nueua orden mia porque estos negocios de entradas sino $\mathrm{Vm}$ con mucho fundamento no son de efecto, y mtes. demian que aprouechem, y de lo que cercado y lo demas que a V. Sa. le pareciera que deuere prouer sera seruido mandar auisarme". 1 de septiembre de 1582. (BO ABNB, ALP, CACh-35). 


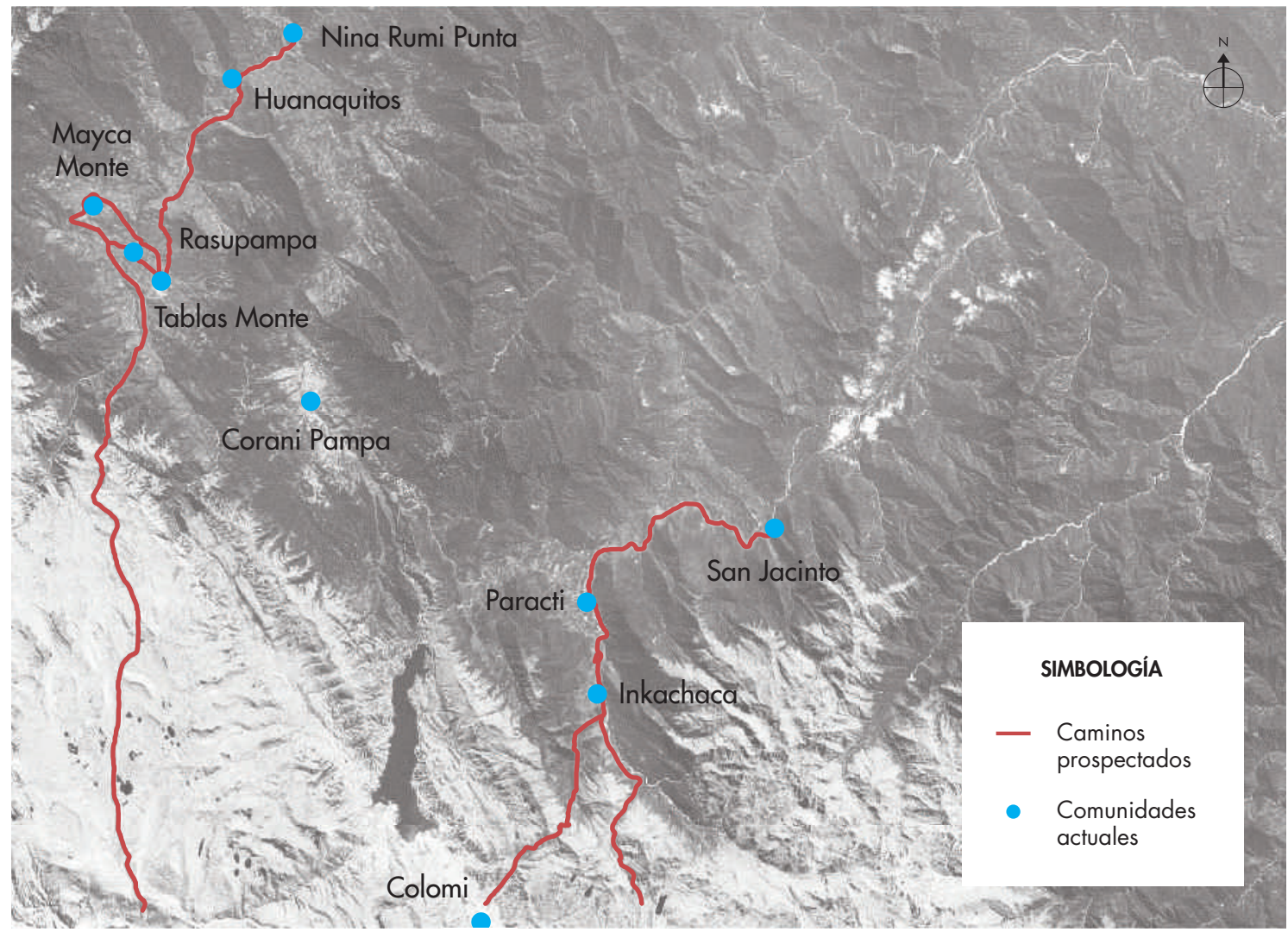

Figura 6. Mapa de caminos de clara factura inca de la puna hacia los yungas ("montaña") de Incachaca y Tablas Monte en Cochabamba. Figure 6. Segment of a paved Inca road along the Colomi-Incachaca branch (photo by Walter Sánchez C.).

Con esta orden se prohíben las licencias de entradas de civiles hacia la montaña de los Moxos desde Cochabamba. No obstante, sabemos que en 1588 Angulo realiza una nueva entrada, al parecer por la puna de Colomi, la que también fracasa (fig. 6).

\section{CONCLUSIONES PRELIMINARES}

Los distintos documentos asociados a la entrada revelan varios elementos. Hay que destacar en primer lugar que Hinojoza realiza sus entradas solo con soldados españoles. Es decir, al no existir mención de que hayan participado gente local de los valles de Cochabamba, menos de los yungas o de la montaña, se estaría refrendando la idea de una no participación consciente, cuyo fin sería no dar a conocer las rutas de ingreso. En segundo lugar, para hacer efectiva esta entrada, tiene que ofrecer, como una autoridad colonial, una repartición de tierras a los soldados como pago, ya que el ingreso supone riesgos. Finalmente, en su ingreso solamente encuentra un pequeño pueblo de pocos habitantes quienes, luego de un pequeño enfrentamiento, huyen; es decir, asumen una táctica constante en los grupos de bosque.

Es destacado cómo la entrada de Francisco de Hinojosa revela la inexistencia de algún camino. Si asumimos que la investigación arqueológica actual ha venido mostrando la presencia de caminos formalmente construidos y que llegan a importantes complejos de presencia inca (cf. Sánchez 2008), existe una fuerte sugerencia de que, durante el Período Colonial temprano, las sociedades de los yungas (sean Umu/Amo/ Yumu, Yuroma o Raché) habrían decidido conscientemente no dar a conocer estas rutas de entradas $y$, posiblemente, cortar esas vías de comunicación a fin de escapar del asedio colonial, aunque manteniendo rutas de comunicación transversales dentro de los yungas y hacia los llanos amazónicas. La decisión consciente 
de clausurar antiguas rutas hacia los valles, habría generado desde entonces una imagen de la montaña de Cochabamba como una zona de frontera infranqueable o solo habitada por mosquitos, serpientes y "leones", hecho que se mantendrá durante todo el Período Colonial y que será refrendada por los padres jesuitas y los curas franciscanos (siglo XVIII y XIX), quienes se esfuerzan por abrir rutas que comuniquen los valles de Cochabamba con las misiones y pueblos ubicados en los llanos de Moxos. Tal imagen contagiará, además, a los actuales investigadores quienes refrendarán este perfil de "frontera" o de zona vacía, generando una visión que no da cuenta de la realidad prehispánica: las constantes interacciones y articulaciones entre las tierras bajas y las tierras altas.

No obstante, si bien muchos caminos Incas fueron abandonados por las mismas comunidades de los yungas durante el periodo colonial, varios ramales siguieron siendo usados como rutas de intercambio (de cereales, papa, fruta, sal, miel, madera, coca) entre comunidades. Tal hecho era evidente incluso hasta finales del siglo $\mathrm{xx}$, cuando seguían bajando tropas de llamas desde las punas de Pocona, Tiraque, Totora, Colomi, Palqa, hacia los yungas de Tablas Monte/San Julian e Incachaca, Arepucho, Chuquiuma y Vandiola.

\section{Archivos consultados}

$\mathrm{ANB}=$ Archivo Nacional de Bolivia

AHMC $=$ Archivo Histórico Municipal de Cochabamba.

\section{NOTAS}

${ }^{1}$ La documentación del siglo XVI y XVII se refiere a los yungas como zona de "montaña".

${ }^{2}$ Los campesinos de la zona de Tablas Monte pronuncian la palabra ñan como yan.

${ }^{3}$ Nacido en España. Posiblemente haya estado emparentado con el General Pedro de Hinojosa, quien dirigió las falanges españolas, diezmadas en las contiendas armadas en la zona de Charcas (Urquidi 1949: 49).

${ }^{4}$ Es importante notar esta "tropa" de 300 llamas que iban de Cochabamba a Potosí llevando harina de maíz. Muestra, fuera del intenso tráfico comercial entre ambas Villas, la importante presencia de este camélido en los valles mesotérmicos.

${ }^{5}$ Voz caída en desuso, pero frecuente en la colonia temprana. Según los contextos de documentos tempranos, puede ser traducido por pelea, lucha, motín, algazara.

\section{REFERENCIAS}

Alconini, S., 2013. Los incas y los guaraníes: conflicto, etnogénesis y fluidez social en la fortaleza de Cuzcotuyo, frontera inca sur-oriental. Arqueoantropológicas 3: 11-26.

Alconini, S., (Ed.), 2016. Entre la vertiente tropical y los valles. Sociedades regionales e interacción prehispánica en los Andes centro-sur. Sucre: The University of Texas-Universidad Real y Pontificia de San Francisco Xavier-Plural.

Brockington, D.; D. Pereira, R. Sanzetenea \& M. A. MuÑoz, 1995. Conchupata: un panteón formativo temprano en el valle de Mizque, Cochabamba, Bolivia. Cochabamba: Universidad Mayor de San Simón.

CÉspedes, R., 2001. Les vallées de Cochabamba sous la domination de Tiahuanacu. Dossier d'Archéologie (Tiahuanacu une civilisation des Andes) 261: 42-49.

Cruz, P., 2014. Fronteras difusas. Nuevas perspectivas en la relación Andes-tierras bajas en tiempos del Inca. En Ocupación inca y dinámicas regionales en los Andes (siglos XV-XVII), C. Rivera, Ed., pp. 155-176. Lima-La Paz: IFEA-Plural.

Del Río, M., 2011. Caciques, territorios y multietnicidad en la frontera oriental: Pocona y Totora en el siglo xvi. Arqueoantropológicas 1: 99-118.

JimÉNEZ DE LA EsPADA, M., (Ed.), 1965. Relaciones geográficas de Indias. Biblioteca de Autores Españoles, Tomos cLXXXıII a CLXXxv. Madrid: Atlas.

Maurtúa, V., (Ed.), 1906. Juicio de límites entre el Perú y Bolivia. Barcelona: Henrich \& Comp.

Mendoza, D., 1976 [1665]. Chronica de la Provincia de San Antonio de los Charcas. La Paz: Casa Municipal de la Cultura.

Pärssinen, M. \& A. SiIRIÄnen, 2003. Andes orientales y Amazonía occidental. Ensayos entre la historia y la arqueología de Bolivia, Brasil y Perú. La Paz: Cima-umsa-Colegio de Historiadores de Bolivia.

Pereira, D. \& R. CÉspedes, 1982. Culturas pre-incas y redes viales en Cochabamba. Cochabamba: INIAM-UMSs.

SAIGNES, T., 1985a. El piedemonte entre los mitos indígenas y las quimeras europeas. En Los Andes orientales: historia de un olvido, T. Saignes, Ed., pp. 33-89. Cochabamba-La Paz: IFEA-CERES.

SAIGNES, T., 1985b. Los Andes orientales: historia de un olvido. Cochabamba-La Paz: IFEA-CERES.

SÁnchez, W., 2008. Inkas "flecheros" y mitmaqkuna. Cambio social y paisajes culturales en los valles y en los yungas de Inkachaca/Paracti y Tablas Monte (Cochabamba-Bolivia, siglos $\mathrm{XV}$-XVI). Uppsala: Uppsala Universitet.

SÁncheZ, W., 2011. Redes viales y entramados relacionales entre los valles, la puna y los yungas de Cochabamba. En En ruta, arqueología, historia y etnografía del tráfico sur andino, L. Núñez \& A. Nielsen, Eds., pp. 177-198. Córdoba: Encuentro Grupo Editor.

SÁNCHEZ, W., 2012. Cruzando paisajes, transitando caminos. El ramal inca de Sipe Sipe hasta Incachaca (Cochabamba, 
Bolivia). En The past ahead. Language, culture and identity in the neotropics, C. Isendahl, Ed., 207-228. Uppsala: Uppsala Universitet.

Sarmiento de Gamboa, P., 1943 [1572]. Historia general de los Incas. Buenos Aires: EMECÉ.

Schramm, R., 2012. Hombres de agua: ¿un rasgo común de las etnias precoloniales en los valles cochabambinos? Arqueoantropológicas 2: 185-194.
Soruco, A. (Ed.), 1899. Digestos y ordenanzas. Acuerdo y decretos de la Municipalidad de Cochabamba. Cochabamba: Municipio de Cochabamba.

URQUIDI, J., 1949 [1571]. El origen de la noble villa de Oropeza (Cochabamba). Fundada por el Capitán Gerónimo de Osorio. Comprobación documental. Cochabamba: Imprenta Universitaria. 